University of Wollongong

Research Online

Faculty of Social Sciences - Papers (Archive) Faculty of Arts, Social Sciences \& Humanities

2014

Physiological cross-sectional area of the oblique head of the adductor pollicis is greater than its transverse counterpart: implications for functional testing

Alexander Stamenkovic

University of Wollongong, as530@uowmail.edu.au

Bridget J. Munro

University of Wollongong, bmunro@uow.edu.au

Gregory E. Peoples

University of Wollongong, peoples@uow.edu.au

Follow this and additional works at: https://ro.uow.edu.au/sspapers

Part of the Education Commons, and the Social and Behavioral Sciences Commons

Research Online is the open access institutional repository for the University of Wollongong. For further information contact the UOW Library: research-pubs@uow.edu.au 


\title{
Physiological cross-sectional area of the oblique head of the adductor pollicis is greater than its transverse counterpart: implications for functional testing
}

\author{
Abstract \\ Introduction: Despite structural distinction between the transverse and oblique heads of the adductor \\ pollicis, in vivo testing continues to consider the adductor pollicis as functionally simplistic. As a muscle's \\ architecture is a strong indicator of function, in this study we aimed to determine whether the \\ physiological cross-sectional areas (PCSAs) of both heads were uniform. Methods: Classical, \\ microdissection, and chemical dissection procedures were conducted on 10 cadaveric left hands to \\ determine structural origin and insertions. Architectural measures of muscle length $\left(L_{m}\right)$, muscle weight \\ $\left(W_{m}\right)$, fascicle length $\left(L_{f}\right)$, sarcomere length $\left(L_{s}\right)$, and pennation angle $(\theta)$ were used to calculate PCSA \\ and fascicle length:muscle length ratio $\left(\mathrm{L}_{\mathrm{f}} \mathrm{Lm}\right)$. Results: The oblique head had greater variation in \\ attachments, significantly greater PCSA $(P=0.008)$, and smaller $\mathrm{Lf}: \mathrm{Lm}(P=0.001)$ than its transverse \\ counterpart. Conclusions: Muscle architecture suggests the oblique head has greater potential for force \\ generation, and the transverse has greater potential for joint excursion.

\section{Disciplines} \\ Education | Social and Behavioral Sciences

\section{Publication Details} \\ Stamenkovic, A., Munro, B. J. \& Peoples, G. E. (2014). Physiological cross-sectional area of the oblique \\ head of the adductor pollicis is greater than its transverse counterpart: implications for functional testing. \\ Muscle and Nerve, 49 (3), 405-412.
}


Submitted to MUSCLE AND NERVE

The physiological cross-sectional area of the oblique head of the adductor pollicis is greater than its transverse counterpart: Implications for functional testing.

Alexander STAMENKOVIC, BSc(Hons.), ${ }^{1,2,4}$ Bridget J. MUNRO, PhD ${ }^{2,3}$ and Gregory E. PEOPLES, $\mathrm{PhD}^{4}$

\footnotetext{
${ }^{1}$ Neural Control of Movement Laboratory, School of Health Sciences, Faculty of Science, Medicine \& Health

${ }^{2}$ Biomechanics Research Laboratory, School of Health Sciences, Faculty of Science, Medicine \& Health

${ }^{3}$ ARC Centre of Excellence in Electromaterials Science and Intelligent Polymer Research Institute

${ }^{4}$ Human Anatomy Laboratory, School of Health Sciences, Faculty of Science, Medicine \& Health
}

\author{
University of Wollongong \\ Northfields Avenue \\ Wollongong NSW 2522 \\ AUSTRALIA \\ Address of corresponding author: \\ Dr Gregory E. PEOPLES \\ School of Health Sciences \\ Faculty of Science, Medicine \& Health \\ University of Wollongong \\ Northfields Avenue \\ Wollongong NSW 2522 \\ AUSTRALIA \\ Tel: +61-(02)-4221-5172 \\ Fax: +61-(02)-4221-5945 \\ Email: peoples@uow.edu.au
}

Number of words in abstract: 150

Number of words and characters (with spaces) in text: 4,161 $(28,235)$

Number of Figures: 4

Number of Tables: 1

Running Head: ADDUCTOR POLLICIS AND MUSCLE ARCHITECTURE 
Adductor pollicis and muscle architecture

Page $\mathbf{2}$ of $\mathbf{2 8}$

\begin{abstract}
:
Introduction: Despite structural distinction between the transverse and oblique heads of the adductor pollicis, in vivo testing continues to consider the adductor pollicis as functionally simplistic. As a muscle's architecture is a strong indicator of function, this study aimed to determine whether the physiological cross-sectional areas (PCSA) of both heads were uniform.

Methods: Classical, micro- and chemical dissection procedures were conducted on 10 cadaveric left hands to determine structural origin and insertions. Architectural measures of muscle length $\left(\mathrm{L}_{\mathrm{m}}\right)$, muscle weight $\left(\mathrm{W}_{\mathrm{m}}\right)$, fascicle length $\left(\mathrm{L}_{\mathrm{f}}\right)$, sarcomere length $\left(\mathrm{L}_{\mathrm{s}}\right)$, and pennation angle $(\theta)$ were used to calculate PCSA and fascicle length-to-muscle length ratio $\left(\mathrm{L}_{\mathrm{f}}: \mathrm{L}_{\mathrm{m}}\right)$.

Results: The oblique head had greater variation in attachments, significantly greater PCSA ( $P$ $=0.008)$ and smaller $\mathrm{L}_{\mathrm{f}}: \mathrm{L}_{\mathrm{m}}(P=0.001)$ than its transverse counterpart.

Conclusion: Muscle architecture suggests the oblique head has greater potential for force generation, and the transverse has a greater potential for joint excursion.
\end{abstract}

KEYWORDS: adductor pollicis; physiological cross-sectional area; muscle architecture; muscle function; hand 


\section{Adductor pollicis and muscle architecture}

Page $\mathbf{3}$ of $\mathbf{2 8}$

\section{INTRODUCTION}

The adductor pollicis is a thenar muscle comprised of 2 anatomically discrete heads, the transverse and oblique. The muscle is responsible for adduction of the thumb towards the palm. ${ }^{1-4}$ Classical descriptions originally combined the attachments of the triangular shaped muscle arising from the palmar surface of the third metacarpal and converging on the medial base of the first proximal phalanx and sesamoid bone. ${ }^{1}$ Recently such a view has been updated to include the base of the second and third metacarpals and capitate bone of the distal carpal row, ${ }^{2,3}$ however some texts still retain simplistic attachment information. ${ }^{4}$ This muscle is used commonly to examine skeletal muscle function in the context of muscle fatigue, ${ }^{5}$ chronic heart failure, ${ }^{6}$ force/velocity relationships, ${ }^{7}$ muscle perfusion, ${ }^{8}$ altitude exposure, ${ }^{9}$ task-specific accuracy, ${ }^{10}$ and strength. ${ }^{11}$ The broad scope of experimental designs and fields which utilize the adductor pollicis have been done based upon the simplicity in which testing procedures can be applied. ${ }^{12}$ This can be attributed to 2 main factors: first, the adductor pollicis has a simple, straight-forward action in vivo that allows for optimal force and joint position in a range of daily functions and tasks, including gripping, pinching, and clasping of objects; ${ }^{13,14}$ and second, the adductor pollicis is innervated by only the ulnar nerve. ${ }^{15,16}$ These factors are complemented by the size and position of the muscle. For example, assessing maximal isometric contraction of such a small muscle minimizes the influence of cardiac output on muscle function ${ }^{6}$ and allows for muscle assessment independent of systemic influences. Adductor pollicis is also not as susceptible to disuse atrophy like other large muscle groups used for testing skeletal muscle contractility and fatigue, reaffirming its utility for assessing skeletal muscle function. ${ }^{17}$

While research into the functional capacity of specific muscles has traditionally biased methods such as fiber typing, ${ }^{18}$ this has not been sufficient to explain the functional role of the adductor pollicis. In fact, there is a large body of evidence that suggests the 


\section{Adductor pollicis and muscle architecture}

Page $\mathbf{4}$ of $\mathbf{2 8}$

arrangement and placement of a muscle, or its 'muscle architecture,' is one of the strongest determinants of its function. ${ }^{18-20}$ Architectural variables of physiological cross-sectional area (PCSA), muscle length $\left(\mathrm{L}_{\mathrm{m}}\right)$, and fascicle length $\left(\mathrm{L}_{\mathrm{f}}\right)$, quantified as fascicle length-to-muscle length ratio $\left(\mathrm{L}_{\mathrm{f}}: \mathrm{L}_{\mathrm{m}}\right)$ have been used to indicate particular functional design characteristics such as force generation or joint excursion. ${ }^{18}$ When these variables are compiled for a range of skeletal muscles across the human body (Figure 1) an inverse relationship can be seen, especially for muscles of the lower limb. Values closer to 1 for $\mathrm{L}_{\mathrm{f}}: \mathrm{L}_{\mathrm{m}}$ (Figure 1: Quadrant 1) depict muscles with longer fascicle lengths spanning the entire length of the muscle and are followed by lower PCSA values, indicating a higher capacity for joint excursion. Those muscles with higher PCSA and lower $\mathrm{L}_{\mathrm{f}}: \mathrm{L}_{\mathrm{m}}$ values, represented in Quadrant 4, conversely have a greater potential for force generation.

\section{INSERT FIGURE 1 ABOUT HERE}

The concept of muscle architecture ${ }^{19}$ for the adductor pollicis has been considered previously. ${ }^{21,22}$ The rudimentary determination of fascicle length ${ }^{21}$ or lack of statistical analysis and complementary gross structural information, ${ }^{22}$ however, have made it difficult to infer functional implications from the published datasets. This highlights the need for a more systematic approach to architectural analysis of this muscle if predictive models are to be derived from the results. Classically, such an approach has been demonstrated in studies of lower limb muscle architecture, ${ }^{23}$ where comparisons to more recent data give errors in architectural measures of up to $200 \%{ }^{24}$ Consideration of attachment sites and placement within the body are crucial, as the sensitivity of comparing muscle architecture decreases due to regional variability. ${ }^{24-26}$ Therefore, although muscle architecture is a strong determinant of contractility and muscle function, results must be considered with respect to gross structure, as the topography of a muscle gives insight into the constraints placed upon a muscle. This is apparent for the smaller muscles of the forearm (Figure 1: Quadrant 3) which share similar 


\section{Adductor pollicis and muscle architecture}

Page $\mathbf{5}$ of $\mathbf{2 8}$

PCSA yet vary in their joint excursion ability ${ }^{27}$ and, in the case of the adductor pollicis (Figure 1: Quadrant 1), shows a design trait which conflicts with its published functional findings. ${ }^{13,14,28}$ In fact, simply grouping the 2 heads of the muscle, transverse and oblique, might further distort the qualities of muscle architecture and our understanding of its functional role.

Although the adductor pollicis is a muscle with simple functional properties, it has not been determined whether the architectural parameters of both the transverse and oblique heads reflect this uniformity. This is because current assumptions of anatomy and architecture are often oversimplified and generic, yet the specific architecture of muscles has long been considered a vital, ${ }^{18}$ if not the most important determinant of function. ${ }^{19,20}$ A lack of research of the separate heads of the adductor pollicis, compounded with the variability of results in the literature for this muscle, ${ }^{21,22}$ emphasize the need for structural and architectural analyses to be conducted prior to use of this muscle in functional testing. Hence, the purpose of this study was to systematically analyze the muscle architecture of the adductor pollicis and determine whether the architecture is represented uniformly within both the transverse and oblique heads.

\section{MATERIALS AND METHODS}

According to recent recommendations, ${ }^{26}$ anatomical procedures, including classical, micro- and chemical dissection, and an adequate sample size were used to describe and quantify measures of muscle architecture of the transverse and oblique heads of the adductor pollicis. These architectural parameters were then input into formulae $e^{29,30}$ to calculate variables of functional significance to determine the implications of structure on muscle 


\section{Adductor pollicis and muscle architecture}

Page 6 of $\mathbf{2 8}$

function. All procedures were approved by our institutional Human Research Ethics Committee (HE11/040) in accordance with the Declaration of Helsinki, 1975.

The left hands of 10 embalmed and perfused cadavers ( 7 male, 3 female; age $74.5 \pm$ 8.4 years at death; gross hand volume, $437.50 \pm 91.05 \mathrm{~mL}$ ) were selected for architectural determination. Specimens were excluded based upon previous dissection, which may have disrupted the carpal rows, observable disuse atrophy of the hand, and general or specific documentation (from medical history information) pertaining to musculoskeletal abnormalities which may have affected thenar function (e.g. osteo- or rheumatoid arthritis).

Hand volume was measured using an adapted water displacement technique ${ }^{31}$ to characterize overall hand size of the 10 specimens and to compare it to normative anthropological data. ${ }^{32,33}$ Specimens were lowered into a filled vessel so that submersion of the hand would cause excess water to flow through an outlet valve into a capture volume container. The captured volume was then weighed (0.0001 g - Adventurer Pro, Ohaus, Australia) and converted from grams to milliliters to determine gross hand volume. The apparatus was calibrated using plastic spheres of known volume, and a test-retest design was implemented to confirm reliability of the technique $\left[\operatorname{ICC}_{(2,1)}=0.999\right]$.

\section{Classical Dissection:}

Initial dissection involved removal of skin, subcutaneous fat, and fascia covering the palmar and thenar regions of the hands. The sheaths of the tendons of flexor pollicis longus, flexor digitorum superficialis, and flexor digitorum profundus were cleaned and dissected out at the metacarpo-phalangeal joints. Then, together with the insertions of the lumbrical muscles, the tendons were pulled through the carpal tunnel and flexor retinaculum to increase the viable working space for identification of the heads of the adductor pollicis.

To properly identify key origin and insertion information, the muscles making up the thenar eminence were excised. As only attachments that required sharp (or bladed) dissection 


\section{Adductor pollicis and muscle architecture}

Page $\mathbf{7}$ of $\mathbf{2 8}$

were considered legitimate origins or insertions, ${ }^{34}$ adhesions to other structures that could be removed through blunt dissection were conducted under a stereo-dissection microscope at $5 \mathrm{x}$ magnification (SMZ800, Nikon, Australia). Origin and insertion information allowed for correct differentiation of the adductor pollicis from tightly adhering fibers of the deep head of flexor pollicis brevis. ${ }^{35}$ The deep palmar arch was pinned and used as an anatomical landmark ${ }^{34}$ to clearly define the heads of the adductor pollicis. Fibers distal to the arch were considered to be part of the transverse head, and fibers proximal to the arch were considered to form the oblique head. Each origin and insertion point was pinned with the muscle attachments undisturbed and recorded immediately after the muscle was removed to incorporate underside attachments in preparation for micro-dissection and architectural measurements.

\section{Micro-dissection:}

Dissection methods described for intrinsic hand muscles ${ }^{30}$ were followed so that architectural measures maintained consistency with the literature. To ensure the accuracy of future measurements, the excised adductor pollicis was cleaned of external tendon and fascia to the level of the muscle under 25x magnification (SMZ800, Nikon, Japan). The muscle was divided (based upon criteria mentioned previously) into its separate transverse and oblique heads, and architectural measurements were taken a minimum of 3 times with a mean value being calculated for analysis.

Muscle lengths $\left(\mathrm{L}_{\mathrm{m}}, \mathrm{mm}\right)$ were measured under a stereo-dissection microscope at $5 \mathrm{x}$ magnification (SMZ800, Nikon, Japan) from the most proximal portion of the muscle belly to the most distal portion using anatomical grade dial Vernier calipers (Mitutoyo, Japan) to the nearest $0.02 \mathrm{~mm}$. As the hydration status of formalin-fixed tissue can offset calculations of PCSA by up to $9 \%$, saturation was also required to mimic that of in vivo muscle. ${ }^{36}$ Muscle heads were soaked in a $20 \%$ rehydrating solution (Hydro, Genelyn, Australia) for 24 hours to 


\section{Adductor pollicis and muscle architecture}

Page $\mathbf{8}$ of $\mathbf{2 8}$

obtain accurate wet weight measures $\left(\mathrm{W}_{\mathrm{m}}, \mathrm{g}\right)$. After saturation, specimens were left to stand on absorbent paper, rotated every 3 minutes, and then allowed to air dry for 10 minutes to remove residual water on the surface of the muscle. Each muscle head was then weighed on a standardized and calibrated electronic scale (Adventurer Pro, Ohaus, Australia) accurate to 0.0001 g. Muscle volume $\left(\mathrm{V}_{\mathrm{m}}, \mathrm{mL}\right)$ was derived by water displacement, ${ }^{30}$ wherein a $100 \mathrm{~mL}$ beaker was filled to capacity and weighed (Adventurer Pro, Ohaus, Australia), and the muscle was gently lowered into the beaker while allowing excess water to overflow. The difference between beaker pre- and post-weight values $(\mathrm{g})$ was taken and converted to milliliters for final muscle volume determination. As fascicle length has been shown to be indicative of fiber length, ${ }^{29}$ three fascicle bundles, comprised of approximately 50 fibers each $^{37}$ were removed, and fascicle length $\left(\mathrm{L}_{\mathrm{f}}, \mathrm{mm}\right)$ was measured to the nearest $0.02 \mathrm{~mm}$ of muscle length under a stereo-dissection microscope (SMZ800, Nikon, Japan) at 25x magnification. Coefficients of variation were calculated for all specimens to determine whether variations between specimens were consistent for the specified region of the hand. ${ }^{26}$

\section{Chemical Dissection:}

Chemical dissection was utilized to remove the dense connective tissue of the perimysium without damaging the muscle fibers encapsulated within. Based on pilot testing and previously published techniques, ${ }^{24,38}$ fascicles were placed into a $20 \% \mathrm{v} / \mathrm{v}$ nitric acid solution for 72 hours with stirring for 30 minutes within every 24-hour cycle to prevent stagnation of the acid. After acid digestion, fascicles were rinsed consecutively in 3 distilled water baths, mounted onto glass slides, and placed under a light microscope (ML2000, Meiji, Japan). Sarcomere banding was visualized at 400x magnification to quantify sarcomere length, whereby, consistent with the literature, sarcomere measures were quantified from the proximal, middle, and distal regions of each fascicle. ${ }^{24,27,29,30}$ An optical reticle calibrated at 400x magnification (ML2000, Meiji, Japan) was used as a standardized measurement tool, 


\section{Adductor pollicis and muscle architecture}

Page $\mathbf{9}$ of $\mathbf{2 8}$

and sarcomeres were counted within each region to sufficiently calculate a mean sarcomere length $\left(\mathrm{L}_{\mathrm{s}}\right)$ for each fascicle. ${ }^{39}$ These values were used to normalize fascicle lengths to an optimal length ( $\mathrm{L}_{\text {fopt}}$; Equation 1).

$$
\mathrm{L}_{\text {fopt }}=\mathrm{L}_{\mathrm{f}} \cdot \frac{\mathrm{L}_{\text {sopt }}}{\mathrm{L}_{\mathrm{s}}}
$$

\section{Equation 1}

where: $\mathrm{L}_{\text {fopt }}=$ optimal fascicle length $(\mathrm{mm})$

$$
\begin{aligned}
& \mathrm{L}_{\mathrm{f}}=\text { fascicle length }(\mathrm{mm}) \\
& \mathrm{L}_{\text {sopt }}=\text { optimal mammalian sarcomere length }(2.64 \mu \mathrm{m})^{40} \\
& \mathrm{~L}_{\mathrm{s}} \quad=\text { sarcomere length }(\mu \mathrm{m})
\end{aligned}
$$

Whole muscle heads were placed into a solution of $25 \%$ formic acid for 6 hours to remove tightly-adhering fascia and gain a clearer understanding of the pennate nature of the adductor pollicis. Pennation angle $\left(\theta,^{\circ}\right)$ was measured under a stereo-dissection microscope (SMZ800, Nikon, Japan) at 5x magnification using built-in, calibrated image software (Nikon Elements, Nikon, Japan) similar to methods described previously for the intrinsic foot muscles. ${ }^{41}$ To allow comparisons to the literature, ${ }^{22,27,29,30}$ software was also calibrated to a standard goniometer (E-Z Read, JAMAR, Australia). Briefly, the distal tendon was traced and pinned along the proximal, middle, and distal portions of the muscle head, and a line was traced along the visible muscle fascicle. Three measures were taken in each region to allow for a global representation of the muscle. In order to apply pennation angle data into future measures of functional variables, it was necessary to normalize raw pennation values to an optimal position similar to fascicle length. ${ }^{30}$

Functional variables of PCSA (Equation 2) and $\mathrm{L}_{\mathrm{f}}: \mathrm{L}_{\mathrm{m}}$ were calculated using the relevant architectural inputs.

$$
\operatorname{PCSA}=\frac{W_{m} \cdot \cos \theta}{\rho \cdot L_{\text {fopt }}}
$$


Adductor pollicis and muscle architecture

Page $\mathbf{1 0}$ of $\mathbf{2 8}$

$$
\text { where: } \begin{aligned}
\text { PCSA } & =\text { physiological cross-sectional area }\left(\mathrm{cm}^{2}\right) \\
\mathrm{W}_{\mathrm{m}} & =\text { muscle wet weight }(\mathrm{g}) \\
\theta & =\text { pennation angle }\left(^{\circ}\right) \\
\rho & =\text { muscle density }\left(\text { in vivo }=1.057 \mathrm{~g} / \mathrm{cm}^{3}\right)^{42} \\
\mathrm{~L}_{\mathrm{fopt}} & =\text { optimal fascicle length }(\mathrm{mm})
\end{aligned}
$$

\section{Statistical Analysis:}

Qualitative data for origin and insertion points and muscle classification were tabulated on a case-by-case basis and recorded as frequencies. Architectural measures of muscle length, muscle weight, muscle volume, optimal fascicle length, and sarcomere length, as well as the calculated functional variables of PCSA and $\mathrm{L}_{\mathrm{f}}: \mathrm{L}_{\mathrm{m}}$ were recorded, tabulated, and expressed as means and standard deviations. Statistical analysis software (Statistix, Version 8, Tallahassee, Florida, USA) was used for a within-subject design for the separate architectural and calculated functional measures of both the transverse and oblique heads of the adductor pollicis. Paired $t$-tests were conducted to determine whether the architecture of the transverse head differed from that of the oblique head, with significance set at $P<0.05$.

\section{RESULTS}

\section{Muscle Origin/Insertion Variation:}

The origin and insertion points for the transverse and oblique heads of the adductor pollicis can be visualized in Figure 2. The transverse head showed less variation than its oblique counterpart and always originated from the lateral or palmar shaft of the third metacarpal bone (100\% observed). Additional attachments to the palmar aponeurosis were also noted for the transverse head, most often at the most distal portion of the muscle $(40 \%$ observed). The oblique head was most frequently observed to originate from the base of the 


\section{Adductor pollicis and muscle architecture}

Page $\mathbf{1 1}$ of $\mathbf{2 8}$

third metacarpal (100\% observed) and the palmar carpo-metacarpal ligaments associated with the trapezoid and capitate carpal bones (90\% observed). Shorter attachment sites to the articulating second metacarpal bone and the corresponding metacarpal ligaments between the second and fourth metacarpals were also prevalent ( $90 \%$ observed). Three specimens (30\% observed) attached as laterally as the trapezium carpal bone, while a further specimen $(10 \%$ observed) was seen to extend completely over to the base of the fourth metacarpal.

As the muscle heads share a common distal tendon, insertion information was reported as a single site for both the transverse and oblique heads. This remained highly consistent, with $100 \%$ of specimens inserting onto the medial aspects of the head of the first metacarpal head, base of the first proximal phalanx, and medial sesamoid bone of the metacarpophalangeal joint.

\section{INSERT FIGURE 2 ABOUT HERE}

\section{$\underline{\text { Architectural Measures: }}$}

Table 1 shows significant architectural differences between the transverse and oblique heads of the adductor pollicis. The oblique head had a significantly larger muscle weight and length compared to the transverse head. Optimal fascicle lengths however, showed a contrary relationship, with the transverse fascicles spanning a significantly longer distance of the muscle belly compared to the oblique fascicles. Despite a gross $20 \%$ difference between the transverse and oblique heads, there was no significant difference for muscle volume. Sarcomere length and optimal pennation angles were also similar between muscle heads.

\section{INSERT TABLE 1 ABOUT HERE}

\section{Functional Variables:}

As expected with differences found between the heads in architectural measures, PCSA and $\mathrm{L}_{\mathrm{f}}: \mathrm{L}_{\mathrm{m}}$ for the muscle heads also differed significantly (Table 1). In fact, the PCSA 


\section{Adductor pollicis and muscle architecture}

Page 12 of 28

values for the oblique head were nearly $60 \%$ greater than that of the transverse head, while conversely the transverse head displayed significantly greater $\mathrm{L}_{\mathrm{f}}: \mathrm{L}_{\mathrm{m}}$ compared to the oblique head.

\section{DISCUSSION}

We analyzed systematically the architecture of the 2 heads of the adductor pollicis and determined that measures of muscle weight and muscle length were significantly greater and fascicle length significantly smaller in the oblique head than its transverse counterpart (Figure 3). Furthermore, measures of functional significance, PCSA and $\mathrm{L}_{\mathrm{f}}: \mathrm{L}_{\mathrm{m}}$, were also found to differ significantly indicating that the transverse head has a greater penchant for muscle joint excursion and the oblique head, for force generation within the surrounds of the hand and forearm region.

\section{INSERT FIGURE 3 ABOUT HERE}

From a global perspective, the variability of attachments for the transverse and oblique heads of the adductor pollicis, while great, remain within the confines of previously reported structural information. ${ }^{15,34,43}$ This is complemented by the quantifiable architectural variability deduced for fascicle length expected in the hand region ${ }^{26}$ and anthropometrically, by the overall size of specimens lying within ranges reported previously. ${ }^{32,33}$ These factors give strong evidence for the normalcy of specimens in this investigation.

The detailed collection and analysis of the gross structure undertaken ensure that a true representation of the adductor pollicis muscle is understood and hence, will reflect accurate muscle architectural analysis. Furthermore, based upon the variation found within the gross structure of the muscle heads in both origin and insertion attachments it should be expected that the architecture will also differ between the 2 heads. This is especially apparent for the oblique head, which was found to originate anywhere from the base of the fourth 


\section{Adductor pollicis and muscle architecture}

Page $\mathbf{1 3}$ of $\mathbf{2 8}$

metacarpal to the trapezium carpal bone and as distal as the midline of the second metacarpal. Natural variability through the use of human cadavers should be expected. ${ }^{44}$ In this study, the degree to which both structural and architectural elements of data collection has been undertaken is considered to be more accurate than previous studies. ${ }^{21,22}$

By following well-documented procedures for determination of muscle architecture, ${ }^{22,26,27,30}$ comparisons between other relative musculature in the body are also possible. Figure 4 details the relationship between PCSA and $\mathrm{L}_{\mathrm{f}}: \mathrm{L}_{\mathrm{m}}$ in the functionally relevant surrounds of the hand and forearm. Three key features can be delineated. First, as expected, the inverse trend between $\mathrm{L}_{\mathrm{f}}: \mathrm{L}_{\mathrm{m}}$ and PCSA shows $\mathrm{L}_{\mathrm{f}}: \mathrm{L}_{\mathrm{m}}$ to be indicative of function and a good measure of muscle design tendency. ${ }^{18}$

Looking at the heads of the adductor pollicis separately, they do not aggregate together as would be expected from a homogenous muscle, rather they separate as the aforementioned trend would suggest of muscles with different design tendencies. ${ }^{18}$ An early anatomical study of thumb function ${ }^{45}$ determined that, based on the action of the separate heads of the adductor pollicis, the transverse head was the primary force generator for grip and pinch tasks. The capability for force was attributed to the fiber arrangement of the transverse head being more perpendicular to the metacarpo-phalangeal joint and long axis of the thumb. Based purely on muscle architecture, our results portray a different picture. Greater $\mathrm{L}_{\mathrm{f}}: \mathrm{L}_{\mathrm{m}}$ and larger fascicle lengths allow the transverse head to have greater potential for velocity production and joint excursion as already concluded. This would make better use of its perpendicular arrangement in the initial stages while leaving force generation to the better-suited oblique head and agrees with the mechanical advantage information for the joints of the thumb. ${ }^{46}$ The transverse head of the adductor pollicis generally had much larger moment arms at the metacarpo-phalangeal joint and the carpo-metacarpal joint for both flexion and adduction ${ }^{46}$ and thus, for a given angular change, will require a longer excursion 


\section{Adductor pollicis and muscle architecture}

Page $\mathbf{1 4}$ of $\mathbf{2 8}$

(as given by findings of fascicle length and $\mathrm{L}_{\mathrm{f}}: \mathrm{L}_{\mathrm{m}}$ ). This suggests that, while muscle architecture can give functionally important information about the contractile properties of a muscle, it may also need to be considered within the muscle-joint complex. ${ }^{47-49}$

\section{INSERT FIGURE 4 ABOUT HERE}

Although testing of muscle architecture effects with regards to the muscle-joint complex have not been reported on the joints of the thumb, the relationship between the extensor carpi radialis brevis $\left(\mathrm{ECR}_{\mathrm{B}}\right)$ and extensor carpi radialis longus $\left(\mathrm{ECR}_{\mathrm{L}}\right)$ might help shed light on what is occurring with the adductor pollicis. When the muscle architecture of these synergists are compared (Figure 4) they present design tendencies for force generation and joint excursion, respectively. The $50 \%$ variation in fiber lengths ${ }^{50}$ responsible for this architectural distinction was originally thought to be compensatory in nature. ${ }^{18}$ When sarcomere length tension curves for both muscles in vivo have been constructed, ${ }^{50}$ the range of optimal tension has been found to differ for both muscles such that changes in the musclejoint complex are in fact accentuated. ${ }^{51}$ It was hypothesized that this difference in design traits for synergist muscles was for functional specificity and allowed for force generation to be maintained throughout a range of joint velocities of the wrist. ${ }^{50}$ When the architecture of both heads of the adductor pollicis are collated (Figure 4: $\mathrm{ADP}_{\mathrm{T}+\mathrm{O}}$ ) from this study and considered to be a functional unit, the adductor pollicis occupies an area of the plot which is separate from the intrinsic hand muscles. Previously, a brief determination of adductor pollicis PCSA suggested that the muscle had a similar force-generating capacity as the muscles of the forearm, ${ }^{22}$ and this is corroborated here based on PCSA values alone. However, the placement of the adductor pollicis within Quadrant 2 strengthens the notion that it may be designed to generate high levels of force in spite of high velocities. ${ }^{52}$ Considering the role of the adductor pollicis in pinch and grip strength ${ }^{14,28}$ and its importance in global hand function, ${ }^{13}$ such functional outcomes seem plausible. The identification of architectural 


\section{Adductor pollicis and muscle architecture}

Page $\mathbf{1 5}$ of $\mathbf{2 8}$

specialization within a muscle assumed to be functionally simplistic ${ }^{5-12}$ particularly emphasizes the importance of correct electrode placement during electromyographic studies. As muscle activity is a measure used consistently in examining physiological outcomes, ${ }^{5-12}$ improper placement can severely affect experimental design and procedures. ${ }^{38}$ Specifically for the adductor pollicis muscle, placement within or between heads may result in inaccurate interpretation of contractile data and implied functional application. In fact, electromyography in conjunction with non-invasive imaging techniques have shown successfully that determination of muscle architecture can be assessed within in vivo cohorts for both static and dynamic scenarios. ${ }^{53-58}$ Although information is sparse, ultrasonographic methods in particular have been shown to be both reliable and valid to reflect certain variables of muscle architecture. ${ }^{58}$ However, such investigations also highlight changes to fascicle length during contraction which vary depending on condition and result in a 'curvature' of fascicles. ${ }^{53,55-57}$ Our cadaveric investigation gives a direct measure of muscle architecture and is an essential basis for any future non-invasive ultrasonographic measures of architecture, provided conditions are matched ${ }^{54}$ (static, relaxed muscle). As such, the findings warrant further investigation. For example, similar results found for pennation angle for the heads of the adductor pollicis could simply be dictated by the spatial restraints of the hand itself in a relaxed state. ${ }^{59}$ Whether this is representative of pennation in vivo (as simple theoretical muscle models would suggest ${ }^{60}$ during contraction or is affected by differences in tendon compliance within the muscle tendon unit $^{53,55}$ and variable pennation gearing ${ }^{56}$ between heads (as other muscles have shown) ${ }^{49,53}$ has yet to be determined. Given that the adductor pollicis is popular in physiological skeletal muscle assessment, ${ }^{5-11}$ such knowledge will have a distinct effect on predictions of force generation. ${ }^{61}$

The architectural differences between the heads of the adductor pollicis muscle outlined here also have potential implications within clinical practice. For example, this 


\section{Adductor pollicis and muscle architecture}

Page $\mathbf{1 6}$ of $\mathbf{2 8}$

knowledge could provide informed decision making in implementation and interpretation of hand and pinch grip assessments, as muscle architecture is known to directly affect clinical measures of muscular strength ${ }^{6,11}$ and fatigue. ${ }^{62}$ In light of thumb pinch restoration, especially in tendon transfer surgery, ${ }^{18}$ this investigation provides evidence to support current operative procedures in matching muscles with similar architecture to retain function. ${ }^{63}$ Variations of design tendencies between the heads would also suggest that thumb rehabilitation design should include a range of static and dynamic speed of contractions in order to encourage return to function. In fact, considered as a single functional unit and combined with the ease of experimental setup and correct electromyographic electrode placement, it allows for a skeletal model which has the potential to be customized to varying isometric or iso-velocity testing conditions.

While muscle architecture can better inform the contractile function of a muscle, it may not always reflect the physiologically expressed outcomes of the interaction between muscle and joint as highlighted in the $\mathrm{ECR}_{\mathrm{B}} / \mathrm{ECR}_{\mathrm{L}}$ relationship. ${ }^{50,51}$ Whether this is also the case for the adductor pollicis is uncertain, and further testing is required. This includes consideration of the series or parallel arrangement of motor units within the adductor pollicis, which are not covered in the current investigation yet are pertinent to skeletal muscle function. ${ }^{64}$ However, based on the macroscopic differences between the heads of the adductor pollicis in both gross structure and muscle architecture we can assume that their functional roles are also different. These differences might better inform other studies of contractile function where fiber typing, an intrinsic factor of muscle contractility, ${ }^{12}$ has not been able to explain function. In saying this, the architectural results of the adductor pollicis in the current investigation confirm why contractile force has not corresponded to traditional fiber typing roles when tested. 


\section{Adductor pollicis and muscle architecture}

Conclusion:

The adductor pollicis muscle has been thoroughly expressed within the functional literature for a wide variety of intervention styles and functional testing. When functionally relevant measures of muscle architecture are assessed for the transverse and oblique heads of the adductor pollicis, they differ significantly. Comparisons to relative musculature of the hand indicate that the transverse head has a penchant for velocity production, and the oblique head produces force generation. While further work considering the muscle-joint complex is needed to determine if such architectural differences translate to in vivo functional heterogeneity, these design tendencies only strengthen the role of adductor pollicis as an indicator of muscle function.

\section{ACKNOWLEDGEMENTS}

The authors acknowledge Mrs. Nicole Jancek, Mr. Todd Price and Ms. Nicole Busby at the University of Wollongong Human Anatomy Laboratory for their technical assistance and Ms. Jessica Fay for the anatomical illustrations. 


\section{Adductor pollicis and muscle architecture}

\section{ABBREVIATIONS}

$\mathrm{ECR}_{\mathrm{B}}$ - extensor carpi radialis brevis muscle

$\mathrm{ECR}_{\mathrm{L}}$ - extensor carpi radialis longus muscle

$\mathrm{L}_{\mathrm{f}}-$ fascicle length

$\mathrm{L}_{\mathrm{f}}: \mathrm{L}_{\mathrm{m}}$ - fascicle length-to-muscle length ratio

$\mathrm{L}_{\text {fopt }}$ - optimal fascicle length

$\mathrm{L}_{\mathrm{m}}-$ muscle length

$\mathrm{L}_{\mathrm{s}}$ - sarcomere length

$\rho-$ in vivo muscle density

$\theta$ - pennation angle

$\mathrm{W}_{\mathrm{m}}-$ muscle wet weight 


\section{Adductor pollicis and muscle architecture}

Page 19 of 28

\section{REFERENCES}

1. Gray H, Carter HV. Anatomy, descriptive and surgical: Greenwich Editions; 2003. (Originally published in 1858)

2. Drake RL, Vogl AW, Mitchell AWM, Gray H. Gray's anatomy for students. Churchill Livingstone/Elsevier; 2010.

3. Moore KL, Dalley AF, Agur AMR. Clinically oriented anatomy: Wolters Kluwer Health/Lippincott Williams \& Wilkins; 2010.

4. Martini FH, Timmons MJ, Tallitsch RB. Human anatomy. Pearson Education, Limited; 2006.

5. Edwards RH, Merton PA, Hill DK, Jones DA. Fatigue of long duration in human skeletal muscle after exercise. J Physiol 1977; 272: 769-778.

6. Buller NP, Jones D, Poole-Wilson PA. Direct measurement of skeletal muscle fatigue in patients with chronic heart failure. Br Heart J 1991; 65: 20-24.

7. de Ruiter CJ, Jones DA, Sargeant AJ, De Haan A. The measurement of force/velocity relationships of fresh and fatigued human adductor pollicis muscle. Eur J Appl Physiol 1999; 80: 386-393.

8. Wright JR, McCloskey DI, Fitzpatrick RC. Effects of muscle perfusion pressure on fatigue and systemic arterial pressure in human subjects. J Appl Physiol 1999; 86: $845-851$.

9. Beidleman BA, Cymerman A, Skrinar GS, Fulco CS, Sawka MN, Lewis SF, et al. Intermittent altitude exposures improve muscular performance at 4,300 m. J Appl Physiol 2003; 95: 1824-1832.

10. Poston B, Enoka RM, Enoka JA. Endpoint accuracy for a small and a large hand muscle in young and old adults during rapid, goal-directed isometric contractions. Exp Brain Res 2008; 187: 373-385. 


\section{Adductor pollicis and muscle architecture}

Page $\mathbf{2 0}$ of $\mathbf{2 8}$

11. Bittner EA. Measurement of muscle strength in the intensive care unit. Crit Care Med 2009; 37: S321.

12. Round JM, Chapman SJ, Ward PS, Jones DA, Fodden DL, Edwards RH. The anatomy and fiber type composition of the human adductor pollicis in relation to its contractile properties. J Neurol Sci 1984; 66: 263.

13. Kaufman KR, An K-N, Litchy WJ, Cooney WP, Chao EYS. In-vivo function of the thumb muscles. Clin Biomech 1999; 14: 141-150.

14. Schreuders TAR, Brandsma JW, Stam HJ. The intrinsic muscles of the hand: Function, assessment and principles for therapeutic intervention. Phys Med Rehab Kuror 2006; 16: 1-9.

15. Chang L, Blair WF. The origin and innervation of the adductor pollicis muscle. J Anat 1985; 140: 381-388.

16. Atkins SE, McGrouther DA, Logan B. The deep (motor) branch of the ulnar nerve: A detailed examination of its course and the clinical significance of its damage. J Hand Surg (British \& European Volume) 2009; 34: 47-57.

17. Narici MV, Bordini M, Cerretelli P. Effect of aging on human adductor pollicis muscle function. J Appl Physiol 1991; 71: 1277-1281.

18. Lieber RL, Friden J. Functional and clinical significance of skeletal muscle architecture. Muscle Nerve 2000; 23: 1647-1666.

19. Gans C, Bock WJ. The functional significance of muscle architecture--a theoretical analysis. Ergeb Anat Entwicklungsgesch 1965; 38: 115-142.

20. Burkholder TJ, Fingado B, Baron S, Lieber RL. Relationship between muscle-fiber types and sizes and muscle architectural properties in the mouse hind-limb. J Morphol 1994; 221: 177-190.

21. Linscheid RL, An K-N, Gross RM. Quantitative analysis of the intrinsic muscles of 


\section{Adductor pollicis and muscle architecture}

Page $\mathbf{2 1}$ of $\mathbf{2 8}$

the hand. Clin Anat 1991; 4: 265-284.

22. Jacobson MD, Abrams RA, Fazeli BM, Lieber RL, Raab R, Botte MJ. Architectural design of the human intrinsic hand muscles. J Hand Surg Am 1992; 17A: 804-809.

23. Friederich JA, Brand RA. Muscle fiber architecture in the human lower limb. J Biomech 1990; 23: 91-95.

24. Ward SR, Lieber RL, Eng CM, Smallwood LH. Are current measurements of lower extremity muscle architecture accurate? Clin Orthop Relat Res 2009; 467:1074-1082.

25. Ward SR, Hentzen ER, Eastlack RK, Fithian DC, Burns KA, Lieber RL, et al. Rotator cuff muscle architecture - implications for glenohumeral stability. Clin Orthop Relat Res 2006:157-163.

26. Tuttle LJ, Ward SR, Lieber RL. Sample size considerations in human muscle architecture studies. Muscle Nerve 2012; 45: 743-745.

27. Lieber RL, Fazeli BM, Botte MJ. Architecture of selected wrist flexor and extensor muscles. J Hand Surg (Am) 1990; 15A: 244-250.

28. Long C, II, Conrad PW, Hall EA, Furler SL. Intrinsic-extrinsic muscle control of the hand in power grip and precision handling: An electromyographic study. J Bone Joint Surg Am 1970; 52: 853.

29. Sacks RD, Roy RR. Architecture of the hind limb muscles of cats: Functional significance. J Morphol 1982; 173: 185-195.

30. Infantolino BW, Challis JH. Architectural properties of the first dorsal interosseous muscle. J Anat 2010; 216: 463-469.

31. Sander AP, Miller AC, Hemenway K, Hajer NM. Upper-extremity volume measurements in women with lymphedema: A comparison of measurements obtained via water displacement with geometrically determined volume. Phys Ther 2002; 82: 1201-1212. 


\section{Adductor pollicis and muscle architecture}

Page $\mathbf{2 2}$ of $\mathbf{2 8}$

32. Clauser CE, McConville JT, Young JW. Weight, volume and center of mass of segments of the human body. Technical Report AMRL-TR-69-70

(AD 710 622). Aerospace Medical Research Laboratory, Wright-Patterson Air Force Base, Ohio. 1969

33. Dempster, WT. Space requirements of the seated operator. Technical Report, WADC-TR-55-159 (AD 87 895). Aerospace Medical Research Laboratory, WrightPatterson Air Force Base, Ohio. 1955

34. Witthaut J, Leclercq C. Anatomy of the adductor pollicis muscle: A basis for release procedures for adduction contractures of the thumb. J Hand Surg (British and European Volume) 1998; 23: 380-383.

35. Day MH, Napier JR. The two heads of flexor pollicis brevis. J Anat 1961; 95: 123 130.

36. Ward SR, Lieber RL. Density and hydration of fresh and fixed human skeletal muscle. J Biomech 2005; 38: 2317-2320.

37. Brown SH, Ward SR, Cook MS, Lieber RL. Architectural analysis of human abdominal wall muscles: Implications for mechanical function. Spine 2011; 36: 355 362.

38. Loeb GE, Gans C. Electromyography for experimentalists. Chicago, IL: University of Chicago Press; 1986.

39. Langenderfer J, Jerabek SA, Thangamani VB, Kuhn JE, Hughes RE. Musculoskeletal parameters of muscles crossing the shoulder and elbow and the effect of sarcomere length sample size on estimation of optimal muscle length. Clin Biomech. 2004; 19: $664-670$

40. Walker SM, Schrodt GR. I segment lengths and thin filament periods in skeletal muscle fibers of the rhesus monkey and the human. Anat Rec 1974; 178: 63-81. 


\section{Adductor pollicis and muscle architecture}

Page $\mathbf{2 3}$ of $\mathbf{2 8}$

41. Ledoux WR, Hirsch BE, Church T, Caunin M. Pennation angles of the intrinsic muscles of the foot. J Biomech 2001; 34: 399-403.

42. Mendez J, Keys A. Density and composition of mammalian muscle. Metabolism 1960; 9: 184.

43. Van Sint Jan S, Rooze M. Anatomical variations of the intrinsic muscles of the thumb. Anat Rec 1994; 238: 131-146.

44. Martin DC, McKee NH, Leekam RN, Chow RS, Agur AM, Medri MK, et al. Comparing human skeletal muscle architectural parameters of cadavers with in vivo ultrasonographic measurements. J Anat 2001; 199: 429-434.

45. McFarlane RM. Observations on the functional anatomy of the intrinsic muscles of the thumb. J Bone Joint Surg Am 1962; 44: 1073.

46. Smutz PW, Kongsayreepong A, Hughes RE, Niebur G, Cooney WP, An KN. Mechanical advantage of the thumb muscles. J Biomech 1998; 31: 565-570.

47. Lieber RL, Boakes JL. Sarcomere length and joint kinematics during torque production in frog hindlimb. Am J Physiol Cell Physiol 1988; 254: C759.

48. Hoy MG, Zajac FE, Gordon ME. A musculoskeletal model of the human lower extremity: The effect of muscle, tendon, and moment arm on the moment-angle relationship of musculotendon actuators at the hip, knee, and ankle. J Biomech 1990; 23: $157-169$.

49. Lieber RL, Shoemaker SD. Muscle, joint, and tendon contributions to the torque profile of frog hip joint. Am J Physiol Regul Integr Comp Physiol 1992; 263: R586.

50. Lieber RL, Loren GJ, Friden J. In vivo measurement of human wrist extensor muscle sarcomere length changes. J Neurophysiol 1994; 71: 874.

51. Lieber RL, Ljung BO, Friden J. Intraoperative sarcomere measurements reveal differential musculoskeletal design of long and short wrist extensors. J Exp Biol 1997; 


\section{Adductor pollicis and muscle architecture}

Page $\mathbf{2 4}$ of $\mathbf{2 8}$

200: 19-25.

52. Lieber RL, Blevins FT. Skeletal muscle architecture of the rabbit hindlimb: Functional implications of muscle design. J Morphol 1989; 199: 93-101.

53. Herbert RD, Gandevia SC. Changes in pennation with joint angle and muscle torque: In vivo measurements in human brachialis muscle. J Physiol 1995; 484: 523-532.

54. Narici MV, Binzoni T, Hiltbrand E, Fasel J, Terrier F, Cerretelli P. In vivo human gastrocnemius architecture with changing joint angle at rest and during graded isometric contraction. J Physiol 1996; 496: 287-297.

55. Hodges PW, Pengel LHM, Herbert RD, Gandevia SC. Measurement of muscle contraction with ultrasound imaging. Muscle Nerve 2003; 27: 682-692.

56. Azizi E, Brainerd EL, Roberts TJ. Variable gearing in pennate muscles. PNAS 2008; 105: $1745-1750$.

57. Namburete AIL, Rana M, Wakeling JM. Computational methods for quantifying in vivo muscle fascicle curvature from ultrasound images. J Biomech 2011; 44: 25382543.

58. Kwah LK, Pinto RZ, Diong J, Herbert RD. Reliability and validity of ultrasound measurements of muscle fascicle length and pennation in humans: A systematic review. J Appl Physiol 2013; 114: 761-769.

59. Burkholder TJ, Fingado B, Baron S, Lieber RL. Relationship between muscle-fiber types and sizes and muscle architectural properties in the mouse hindlimb. J Morphol 1994; 221: 177-190.

60. Otten E. Concepts and models of functional architecture in skeletal muscle. Exer Sport Sci Rev 1988; 16: 89-138.

61. Scott SH, Winter DA. A comparison of three muscle pennation assumptions and their effect on isometric and isotonic force. J Biomech 1991; 24: 163-167. 
Adductor pollicis and muscle architecture

62. Miura H, McCully K, Nioka S, Chance B. Relationship between muscle architectural features and oxygenation status determined by near infrared device. Eur J Appl Physiol 2004; 91: 273-278

63. Lee SK, Wisser JR. Restoration of pinch in intrinsic muscles of the hand. Hand Clinics 2012; 28: 45-51.

64. Loeb GE, Pratt CA, Chanaud CM, Richmond FJR. Distribution and innervation of short, interdigitated muscle fibers in parallel-fibered muscles of the cat hindlimb. J. Morphol 1987; 191:1-15. 


\section{Adductor pollicis and muscle architecture}

Page $\mathbf{2 6}$ of $\mathbf{2 8}$

\section{FIGURE LEGENDS}

Figure 1: Scatterplot of functional variables physiological cross-sectional area (PCSA) and fascicle length-to-muscle length ratio $\left(\mathrm{L}_{\mathrm{f}}: \mathrm{L}_{\mathrm{m}}\right)$ for muscles of the upper and lower limbs. Muscles in Quadrant 1 have values closer to 1 for $\mathrm{L}_{\mathrm{f}}: \mathrm{L}_{\mathrm{m}}$, indicating fascicles which span the entire length of the muscle, and lower PCSA values and thus lower capacity for force generation and higher capacity for joint excursion. Conversely, muscles situated in Quadrant 4 (higher PCSA, lower $\mathrm{L}_{\mathrm{f}}: \mathrm{L}_{\mathrm{m}}$ ) have a greater potential for force generation. Due to absolute size differences of the upper and lower limb musculature, architectural values for the smaller upper limb are shifted leftwards, and design tendencies cannot be clearly delineated, emphasizing the importance of gross structure in determination of muscle function from architecture. $\left(\mathrm{Lum}=\right.$ lumbricals; $\mathrm{ECR}_{\mathrm{L}}=$ extensor carpi radialis longus; $\mathrm{DI}_{1}=$ first dorsal interosseous; $\mathrm{ECR}_{\mathrm{B}}=$ extensor carpi radialis brevis; $\mathrm{ECU}=$ extensor carpi ulnaris; $\mathrm{FCU}=$ flexor carpi ulnaris; $\mathrm{ADP}=$ adductor pollicis; $\mathrm{Semi}_{\mathrm{T}}=$ semitendinosus; $\operatorname{Add}_{\mathrm{L}}=\operatorname{adductor}$ longus; Gastroc $_{\mathrm{Lat}}=$ lateral gastrocnemius; $\mathrm{Tib}_{\mathrm{Ant}}=$ tibialis anterior; $\operatorname{Rec}_{\mathrm{Fem}}=$ rectus femoris; Vastus $_{\text {Med }}=$ vastus medialis). (Data compiled from Lieber et al. ${ }^{27}$ and Jacobson et al. ${ }^{22}$ for upper limb muscles and, Ward et al. ${ }^{24}$ for lower limb muscles).

Figure 2: Graphical representation of the origin and insertion information for the transverse and oblique heads of the adductor pollicis. These can be divided into three general configurations: (a) attachments that project onto the distal second metacarpal, (b) attachments that project laterally from the midline of the third metacarpal and (c), attachments in line with standard textbook presentations $(\mathrm{red}=$ transverse head origin, green $=$ oblique head origin, blue $=$ common tendon insertion). 


\section{Adductor pollicis and muscle architecture}

Page $\mathbf{2 7}$ of $\mathbf{2 8}$

Figure 3: Schematic representation of the architectural differences between the transverse and oblique heads of the adductor pollicis muscle for muscle length $\left(\mathrm{L}_{\mathrm{m}}\right)$, pennation angle $(\theta)$, fascicle length $\left(\mathrm{L}_{\text {fopt }}\right)$, and physiological cross-sectional area (PCSA). Relative differences are represented by percentage increase $(\uparrow)$ or decrease $(\downarrow)$ from transverse head values. Figure is not to scale.

Figure 4: Scatterplot of physiological cross-sectional area (PCSA) and fascicle length-tomuscle length ratio $\left(\mathrm{L}_{\mathrm{f}}: \mathrm{L}_{\mathrm{m}}\right)$ of muscles in the hand and forearm compared to the transverse $\left(\mathrm{ADP}_{\mathrm{T}}\right)$, oblique $\left(\mathrm{ADP}_{\mathrm{O}}\right)$, and combined data $\left(\mathrm{ADP}_{\mathrm{T}+\mathrm{O}}\right)$ for the adductor pollicis. N.B Data for the adductor pollicis from this study is denoted by (\#), and data from Jacobson et al. ${ }^{22}$ are denoted by $(*)$. $\left(\right.$ Lum $=$ lumbricals; $\mathrm{FPB}=$ flexor pollicis brevis; $\mathrm{AbP}_{\mathrm{B}}=$ abductor pollicis brevis; $\mathrm{EPB}=$ extensor pollicis brevis; $\mathrm{OPP}=$ opponens pollicis; $\mathrm{ECR}_{\mathrm{L}}=$ extensor carpi radialis longus; $\mathrm{DI}_{1}=$ first dorsal interosseous; $\mathrm{PI}_{2}=$ second plamar interosseous; $\mathrm{ODM}=$ opponens digiti minimi; $\mathrm{DI}_{2}=$ second dorsal interosseous; $\mathrm{AbP}_{\mathrm{L}}=$ abductor pollicis longus; $\mathrm{FCR}=$ flexor carpi radialis; $\mathrm{ECR}_{\mathrm{B}}=$ extensor carpi radialis brevis; $\mathrm{ECU}=$ extensor carpi ulnaris; FCU = flexor carpi ulnaris). (Data compiled from Lieber et al. ${ }^{27}$ and Jacobson et al. $\left.{ }^{22}\right)$. 


\section{Adductor pollicis and muscle architecture}

Page $\mathbf{2 8}$ of $\mathbf{2 8}$

Table 1. Quantitative analysis of architectural measures of the transverse and oblique heads of the adductor pollicis (values represented as mean $\pm \mathrm{SD} ;{ }^{*} P<0.05$ denotes significant difference between muscle heads, $\mathrm{n}=10$ ).

\begin{tabular}{|c|c|c|c|}
\hline Variable $^{\S}$ & Transverse head & Oblique head & $P$-value \\
\hline $\mathrm{W}_{\mathrm{m}}(\mathrm{g})$ & $3.72 \pm 1.86$ & $5.09 \pm 2.76$ & $0.0329 *$ \\
\hline $\mathrm{L}_{\mathrm{m}}(\mathrm{mm})$ & $41.94 \pm 7.52$ & $46.38 \pm 5.63$ & $0.0057^{*}$ \\
\hline $\mathrm{V}_{\mathrm{m}}(\mathrm{mL})$ & $4.98 \pm 2.04$ & $6.02 \pm 2.60$ & 0.0958 \\
\hline $\mathrm{L}_{\mathrm{s}}(\mu \mathrm{m})$ & $2.26 \pm 0.13$ & $2.26 \pm 0.15$ & 0.9477 \\
\hline $\mathrm{L}_{\text {fopt }}(\mathrm{mm})$ & $28.62 \pm 4.72$ & $23.89 \pm 3.07$ & $0.0017^{*}$ \\
\hline$\theta\left(^{\circ}\right)$ & $15.73 \pm 2.89$ & $15.77 \pm 2.39$ & 0.9561 \\
\hline $\operatorname{PCSA}\left(\mathrm{cm}^{2}\right)$ & $1.18 \pm 0.56$ & $2.01 \pm 1.17$ & $0.0078^{*}$ \\
\hline $\mathrm{L}_{\mathrm{f}}: \mathrm{L}_{\mathrm{m}}$ & $0.63 \pm 0.06$ & $0.51 \pm 0.08$ & $0.0009^{*}$ \\
\hline \multicolumn{4}{|c|}{$\begin{array}{l}{ }^{8} \mathrm{~W}_{\mathrm{m}}=\text { muscle wet weight; } \mathrm{L}_{\mathrm{m}}=\text { muscle length; } \mathrm{V}_{\mathrm{m}}=\text { muscle volume; } \mathrm{L}_{\mathrm{s}}=\text { sarcomere length; } \\
\mathrm{L}_{\text {fopt }}=\text { optimal fascicle length; } \theta=\text { pennation angle; PCSA = physiological cross-sectional }\end{array}$} \\
\hline
\end{tabular}




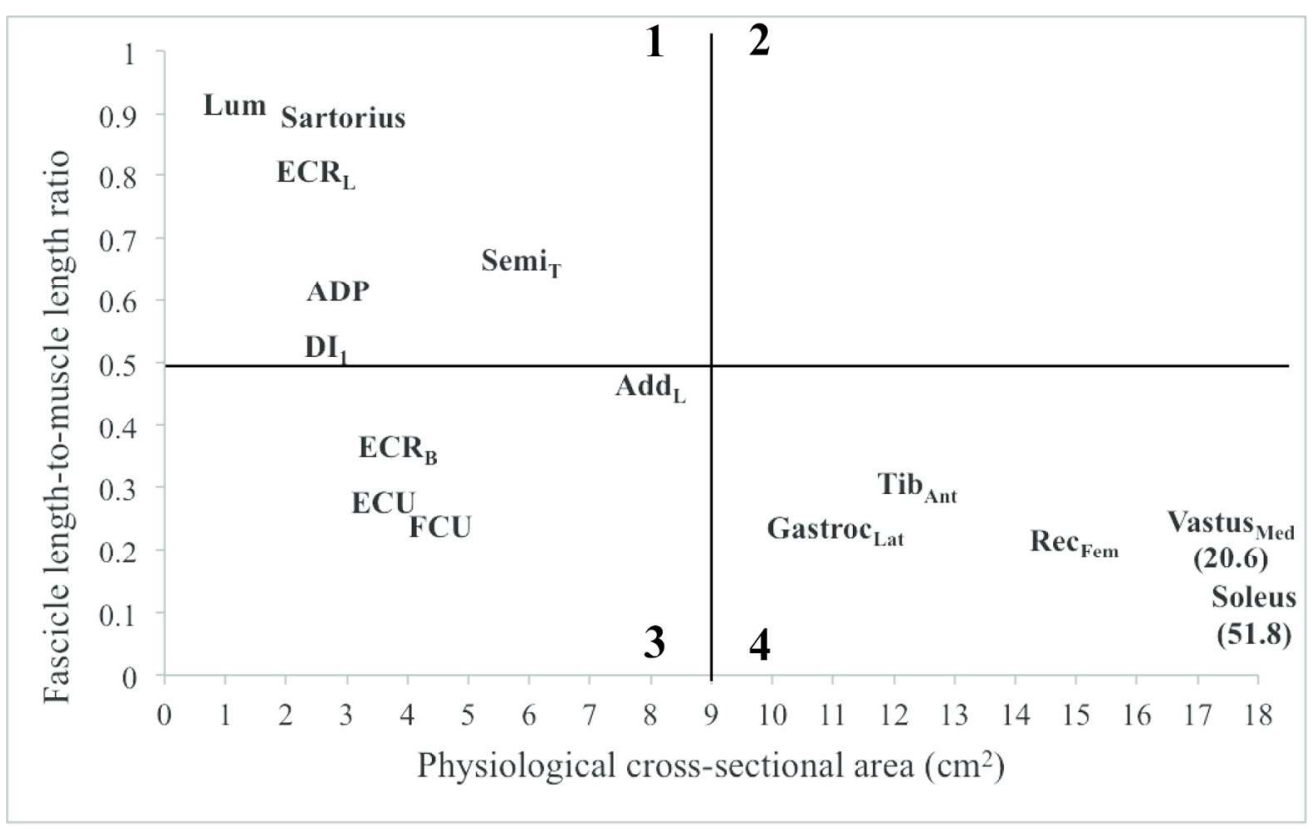

Figure 1: Scatterplot of functional variables physiological cross-sectional area (PCSA) and fascicle length-tomuscle length ratio $\left(L_{f}: L_{m}\right)$ for muscles of the upper and lower limbs. Muscles in Quadrant 1 have values closer to 1 for $L_{f}: L_{m}$, indicating fascicles which span the entire length of the muscle, and lower PCSA values and thus lower capacity for force generation and higher capacity for joint excursion. Conversely, muscles situated in Quadrant 4 (higher PCSA, lower $L_{f}: L_{m}$ ) have a greater potential for force generation. Due to absolute size differences of the upper and lower limb musculature, architectural values for the smaller upper limb are shifted leftwards, and design tendencies cannot be clearly delineated, emphasizing the importance of gross structure in determination of muscle function from architecture. ( $\mathrm{Lum}=$ lumbricals; $E C R_{\mathrm{L}}=$ extensor carpi radialis longus; $\mathrm{DI}_{1}=$ first dorsal interosseous; $\mathrm{ECR}_{\mathrm{B}}=$ extensor carpi radialis brevis; $\mathrm{ECU}=$

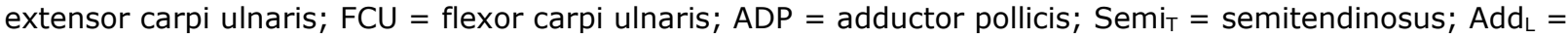
adductor longus; Gastroc $\mathrm{Lat}_{\text {Lat }}=$ lateral gastrocnemius; $\mathrm{Tib}_{\mathrm{Ant}}=$ tibialis anterior; $\mathrm{ReC}_{\mathrm{Fem}}=$ rectus femoris; Vastus $_{\text {Med }}=$ vastus medialis). (Data compiled from Lieber et al. ${ }^{27}$ and Jacobson et al. ${ }^{22}$ for upper limb muscles and, Ward et al. ${ }^{24}$ for lower limb muscles). 


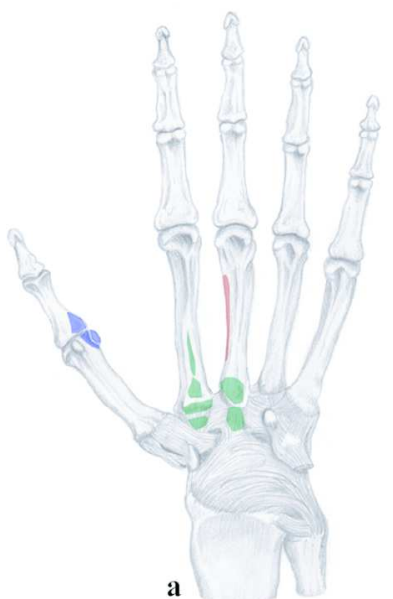

Specimen $1,3,4,7$

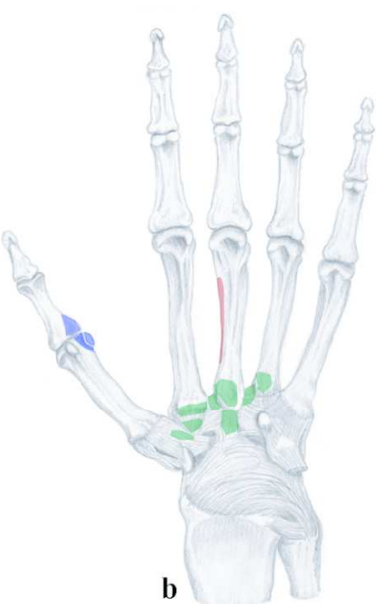

Specimen 2,5,6

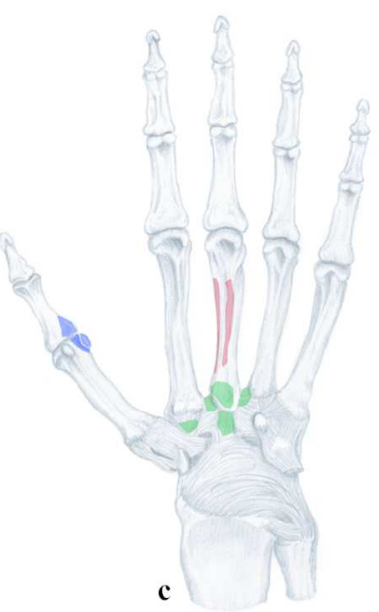

Specimen 8,9,10

Figure 2: Graphical representation of the origin and insertion information for the transverse and oblique heads of the adductor pollicis. These can be divided into three general configurations: (a) attachments that project onto the distal second metacarpal, (b) attachments that project laterally from the midline of the third metacarpal and (c), attachments in line with standard textbook presentations (red = transverse head origin, green $=$ oblique head origin, blue $=$ common tendon insertion). $162 \times 84 \mathrm{~mm}(300 \times 300 \mathrm{DPI})$ 


\section{Transverse Head Oblique Head}

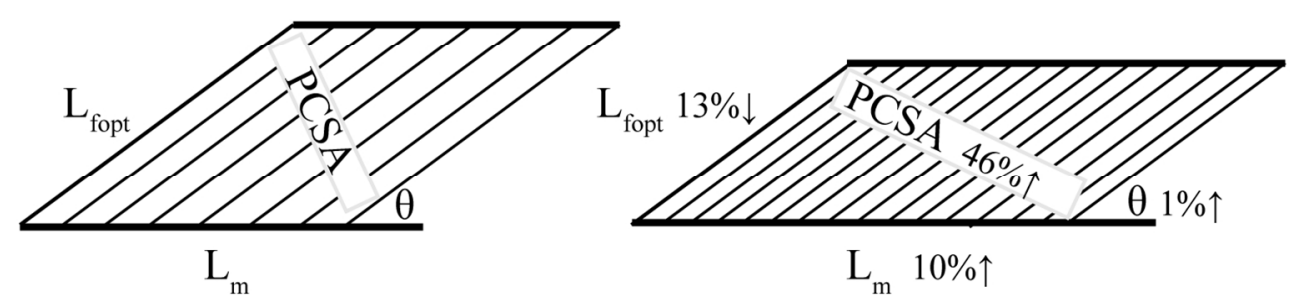

Figure 3: Schematic representation of the architectural differences between the transverse and oblique heads of the adductor pollicis muscle for muscle length $\left(L_{m}\right)$, pennation angle $(\theta)$, fascicle length ( $\left.L_{\text {fopt }}\right)$ and physiological cross-sectional area (PCSA). Relative differences are represented by percentage increase $(\uparrow)$ or decrease $(\downarrow)$ from transverse head values. Figure is not to scale. 


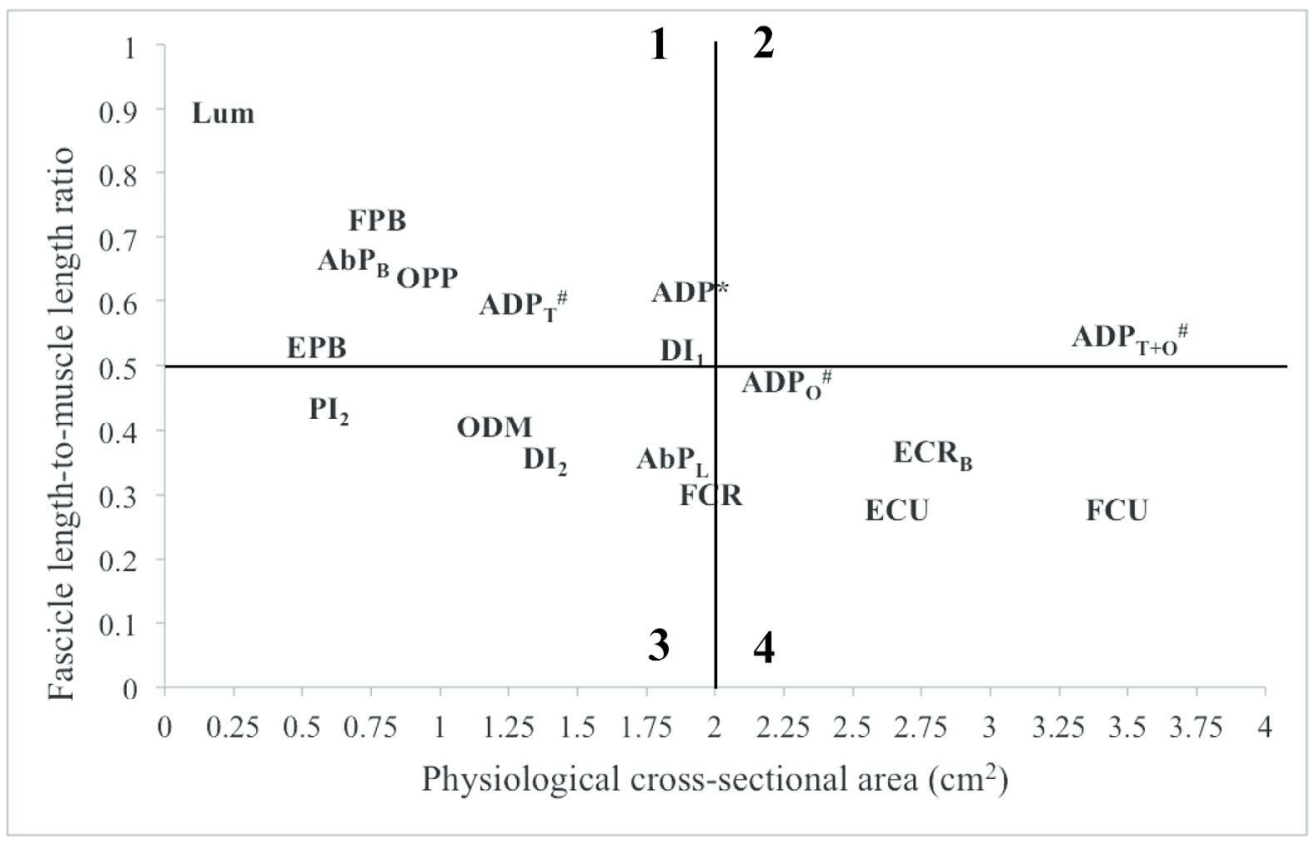

Figure 4: Scatterplot of physiological cross-sectional area (PCSA) and fascicle length-to-muscle length ratio $\left(L_{f}: L_{m}\right)$ of muscles in the hand and forearm compared to the transverse $\left(A D P_{T}\right)$, oblique $\left(A D P_{0}\right)$ and combined data $\left(\mathrm{ADP}_{\mathrm{T}+\mathrm{O}}\right)$ for the adductor pollicis. N.B Data for the adductor pollicis from this study is denoted by $(\#)$ and data from Jacobson et al. ${ }^{22}$ is denoted by $(*)$. (Lum = lumbricals; FPB = flexor pollicis brevis; $\mathrm{AbP}_{\mathrm{B}}=$ abductor pollicis brevis; $\mathrm{EPB}=$ extensor pollicis brevis; OPP = opponens pollicis; $\mathrm{ECR}_{\mathrm{L}}=$ extensor carpi radialis longus; $\mathrm{DI}_{1}=$ first dorsal interosseous; $\mathrm{PI}_{2}=$ second plamar interosseous; ODM = opponens digiti minimi; $\mathrm{DI}_{2}=$ second dorsal interosseous; $A b \mathrm{P}_{\mathrm{L}}=$ abductor pollicis longus; $\mathrm{FCR}=$ flexor carpi radialis; $\mathrm{ECR}_{\mathrm{B}}=$ extensor carpi radialis brevis; $\mathrm{ECU}=$ extensor carpi ulnaris; $\mathrm{FCU}=$ flexor carpi ulnaris). (Data compiled from Lieber et al. ${ }^{27}$ and Jacobson et al. ${ }^{22}$ ).

$$
152 \times 96 \mathrm{~mm}(300 \times 300 \mathrm{DPI})
$$

